\title{
Anemiaceae from Pedra do Elefante, Espírito Santo State, Brazil, with notes on a new hybrid
}

\author{
Nelson Túlio Lage Pena ${ }^{1,3}$, Pedro Bond Schwartsburd ${ }^{2}$ and Anderson Alves-Araújo ${ }^{1}$ \\ Received: 5.12.2016; accepted: 29.09.2017
}

\begin{abstract}
Anemiaceae from Pedra do Elefante, Espírito Santo State, Brazil, with notes on a new hybrid). As part of a floristic survey of the vascular flora of Pedra do Elefante, Espírito Santo State, Brazil, a taxonomic treatment of the Anemiaceae is here presented. Fieldwork was conducted monthly from March 2015 to July 2016. Samples were collected and dried according to the usual methodology and incorporated into the VIC and VIES herbaria. In addition to VIC and VIES, we also studied the specimens form MBML herbarium. Eight species, one variety, and a new hybrid were recorded: Anemia collina, A. × elephantensis nothosp. nov., A. ferruginea var. ferruginea, A. hirsuta, A. luetzelburgii, A. organensis, $A$. patens, A. phyllitidis, A. tomentosa var. tomentosa, and A. tomentosa var. anthriscifolia. Besides the new hybrid, A. tomentosa var. tomentosa is also a new record for the state. Identification key, descriptions, comments, geographical distribution and illustrations are provided for all taxa.
\end{abstract}

Keywords: Anemia, Atlantic Rain Forest, ferns, inselberg, Pteridophytes

RESUMO - (Anemiaceae da Pedra do Elefante, Estado do Espírito Santo, Brasil, com notas sobre um novo híbrido). Como parte do levantamento florístico da flora vascular da Pedra do Elefante, Estado do Espírito Santo, Brasil, é apresentado um tratamento taxonômico da família Anemiaceae. O trabalho de campo foi realizado mensalmente de março de 2015 a julho de 2016. As amostras foram coletadas e secas de acordo com os métodos usuais de coleta botânica e incorporadas aos herbários VIC e VIES. Além dos herbários VIC e VIES, também estudamos os espécimes do herbário MBML. Os herbários MBML, VIC e VIES foram visitados. Oito espécies, uma variedade e um novo híbrido ocorrem na área: Anemia colina, A. ×elephantensis nothosp. nov., A. ferruginea var. ferruginea, A. hirsuta, A. luetzelburgii, A. organensis, A. patens, A. phyllitidis, A. tomentosa var. tomentosa e A. tomentosa var. anthriscifolia. Além do novo híbrido, A. tomentosa var. tomentosa é um novo registro para o Estado. Chave de identificação, descrições, comentários, distribuição geográfica e ilustrações são fornecidas para todos os táxons.

Palavras-chave: Anemia, inselberg, Mata Atlântica, Pteridófitas, samambaias

\section{Introduction}

Anemiaceae is a family of a single genus, Anemia Sw., with 115 species. It is widespread in the Americas, southern Africa, and Madagascar, and has a few species also in India and some oceanic islands (Mickel 2016). In Brazil, 62 species are currently recognized and, among them, 39 occur in the southeastern region (Prado et al. 2015, Mickel 2016, Rabelo \& Schwartsburd 2016). The state of Espírito Santo, which is fully included within the Atlantic Rain Forest Domain, harbors 22 species of Anemia (Prado et al. 2015, Mickel 2016).
The Atlantic Rain Forest is a hotspot of the global biodiversity (Myers et al. 2000) and also an important center of endemism and diversity for ferns and lycophytes (Tryon 1972). Along the Atlantic Rain Forest, especially in southeastern Brazil, rocky outcrop landscapes are very common and show peculiar environmental conditions, such as high isolation, high rates of evaporation, and a discontinuous soil coverage. These features lead to specific plant adaptations and, therefore, generate distinct floristic compositions with high plant endemism rates (Porembski 2000).

Among species of Anemia, hybridization is a wellknown process and has been reported in many studies

1. Universidade Federal do Espírito Santo, Centro Universitário do Norte do Espírito Santo, Rodovia BR 101 Norte, Km 60, $29932-540$ São Mateus, ES, Brasil

2. Universidade Federal de Viçosa, Departamento de Biologia Vegetal, Avenida P.H. Rolfs s.n., 36570-900 Viçosa, MG, Brasil

3. Corresponding author: penatulio@gmail.com 
(Brade 1947, Mickel 1982, 2016, Moran \& Mickel 1995, Mickel \& Smith 2004, Rabelo \& Schwartsburd 2016). Mickel (2016) reported 30 different events of hybridization between different species, and Rabelo \& Schwartsburd (2016) reported another three. Furthermore, Mickel (1982) performed a chromosome counts on Anemia spp. from Mexico. He confirmed a huge ploidy variation ( $2 \mathrm{n}$ to $14 \mathrm{n}$ ) and some unpaired chromosomes (38I to 125I).

As part of a floristic survey of the vascular flora of Pedra do Elefante, state of Espírito Santo, Brazil, we present the taxonomic treatment of Anemiaceae.

\section{Material and methods}

The Área de Preservação Ambiental Pedra do Elefante is located in the municipality of Nova Venécia, Espírito Santo state, Brazil (figure 1) $18^{\circ} 46^{\prime} 30^{\prime \prime} \mathrm{S}$ and $40^{\circ} 27^{\prime} 42^{\prime \prime} \mathrm{W}$. It has an area of 2,562.31 ha with rocky granitic outcrops. The local vegetation type is classified as seasonally semi-deciduous submontane forest (IBGE 2012), with altitudes ranging from 50 to $500 \mathrm{~m}$. The annual precipitation and temperature averages are respectively $800 \mathrm{~mm}$ and $25^{\circ} \mathrm{C}$ (INCAPER 2016).

Fieldwork was performed monthly from March 2015 to July 2016. Specimens were collected and dried according to usual methods for fern collections (Windisch 1992) and then incorporated in VIC and VIES herbaria (Thiers 2016). We also analyzed previously collected specimens from MBML, VIC and VIES herbaria. Classification system follows PPG I (2016) and morphological terms are according to Lellinger (2002) and Mickel (2016).

Information about country, state, and municipality were suppressed from Examined Material: they all refer to "Brazil, Espírito Santo, Nova Venécia" with exception for "additional specimens examined". For the general geographical distribution of the taxa, we consulted Mickel (2016).

\section{Results and Discussion}

A total of 10 taxa of Anemia (eight species, one variety and one newly discovered hybrid) occur in Pedra do Elefante: Anemia collina Raddi, A. $\times$ elephantensis N.T.L. Pena \& Schwartsb. nothosp. nov., A. ferruginea Kunth var. ferruginea, A. hirsuta (L.) Sw., A. luetzelburgii Rosenst., A. organensis Rosent., A. patens Mickel \& Labiak, A. phyllitidis (L.) Sw., A. tomentosa (Sav.) Sw. var. tomentosa, and $A$.

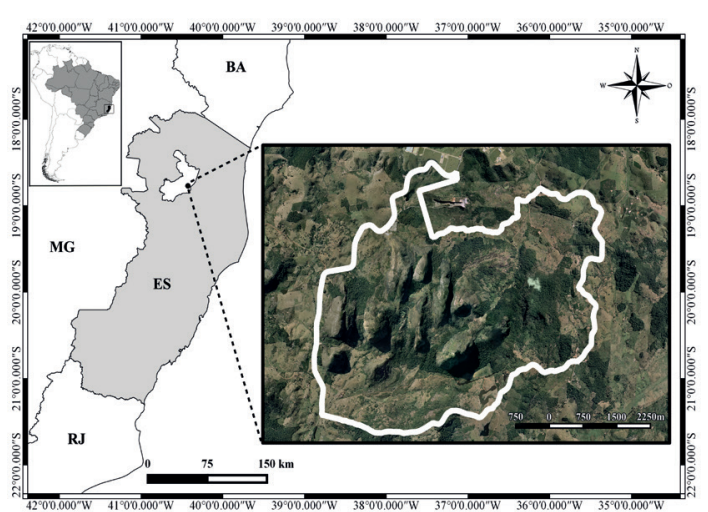

Figure. 1. Location Map of APA Pedra do Elefante, Espírito Santo State, Brazil.

tomentosa var. anthriscifolia (Schrad.) Mickel. The newly described hybrid, Anemia xelephantensis, is believed to be resulted from a cross between $A$. hirsuta and A. luetzelburgii (figures 2-4). Anemia patens has also been recently described by Labiak and Mickel (in Mickel 2016).

Anemia hirsuta is widespread in the Neotropics, and very promiscuous throughout its range. There are reports of hybrids involving $A$. hirsuta and another 12 species (Mickel 2016, Rabelo \& Schwartsburd 2016). Anemia hirsuta and $A$. luetzelburgii belong to the same "Phyllitidis" clade sensu Labiak et al. (2015). Thus, a hybrid between these two species is not surprising. Among the 10 taxa, six are endemic to the Atlantic Forest. The other four are either widespread in the Neotropics or in South America.

New hybrid, Anemia xelephantensis, was not included in the identification key by combining morphological characteristics from both parents. So, a table to distinguish $A$. ×elephantensis and its putative parents is presented.

\section{Taxonomy}

Anemiaceae Link - Plants terrestrial or rupestrial. Rhizomes erect, decumbent or short-creeping, covered by multicellular hairs; hairs filiform to acicular, orange, reddish, brownish or blackish. Fronds hemidimorphic, with the basal pair of pinnae transformed into stalks bearing sporangia, or rarely dimorphic; sterile part of laminae 1-3-pinnate; veins free or rarely anastomosing without included veinlets. Sporangia piriform with apical annulus; spores trilete.

A single genus: Anemia Sw. 
Key to species of Anemia from Pedra do Elefante

1. Laminae 1-pinnate to 1-pinnate-pinnatifid

2. Stipes winged, densely lanose ........................................................................... . collina

2. Stipes not winged, glabrescent to villous

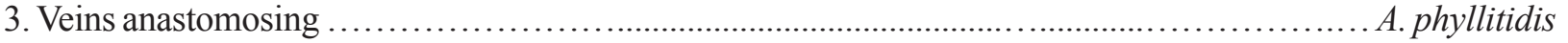

3. Veins free

4. Laminae to $11 \times 4 \mathrm{~cm}$; fertile pinnae always trespassing the sterile laminae portion

A. hirsuta

4. Laminae more than $19 \times 6 \mathrm{~cm}$; fertile pinnae not trespassing the sterile laminae portion ..... A. organensis

1. Laminae 2-pinnate or more dissected

5. Veins proximally free, but anastomosing toward the pinna margins

A. luetzelburgii

5. All veins free

6. Rhizomes with reddish hairs; fertile pinnae 7-11 cm long; basal pinnae 1.5-3 cm long

6. Rhizomes with brownish to orange hairs; fertile pinnae $12-30 \mathrm{~cm}$ long; basal pinnae $\geq 3 \mathrm{~cm}$ long

7. Stipes stramineous; basal pinnae with rounded apex

Anemia patens

7. Stipes dark brown; basal pinnae with acute apex

8. Spores aborted; petioles 27-35 cm long; basal pinnae 8-11 $\times 4-6 \mathrm{~cm}$

A. tomentosa var. tomentosa

8. Spores well-developed; petioles (3-)10-24 cm long; basal pinnae 3-4 × 1-2 cm

A. tomentosa var. anthriscifolia

Anemia collina Raddi, Opusc. Sci. 3. 282. 1819.

Figures 2a,b

Plants terrestrial or rupestrial. Rhizomes erect to decumbent, with orange lanose hairs. Fronds erect, 30-47 cm long; stipes $12-23 \mathrm{~cm} \times 1-2 \mathrm{~mm}$, brownish to greenish, adaxially sulcate, commonly winged, densely lanose; fertile pinnae erect, 22-30 cm long, generally trespassing the sterile laminae portion. Laminae 1-pinnate, ovate, 7-11 pairs of pinnae, apex subconform, 14-18 × 5-10 cm; rachises densely lanose; basal pinnae entire, oblong, margins crenate, apex rounded, 3-5 × 1-2 cm; costae abaxially villous, adaxially sparsely strigose; veins free, strigose on both surfaces. Spores well-developed.

Distribution: Guyana, Suriname and Brazil. In Brazil is reported to northeastern and southeastern (Bahia, Espírito Santo, Minas Gerais, Rio de Janeiro) regions (Mickel 2016).

Specimens examined: estrada para carro em direção a Pedra do Elefante, $18^{\circ} 77^{\prime} 75^{\prime \prime} \mathrm{S}, 40^{\circ} 43^{\prime} 35^{\prime \prime} \mathrm{W}, 330 \mathrm{~m}$, 20-IX-2015, N.T.L. Pena et al. 495 (VIC; VIES); Serra de baixo $18^{\circ} 46^{\prime} 37^{\prime \prime S}, 40^{\circ} 26^{\prime} 38^{\prime \prime} \mathrm{W}, 313 \mathrm{~m}, 18-\mathrm{II}-2008$, P.H. Labiak et al. 4678 (MBML; CEPEC; RB; UPCB).

Comments: Anemia collina is recognized by having densely lanose petioles and rachises, 1-pinnate laminae, rounded pinna apices and crenate margins, and free veins. It differs from $A$. organensis by rhizomes with orange hairs (vs. brownish), commonly winged and densely lanose petioles (vs. not winged and glabrescent to villous), costae abaxially villous and adaxially sparsely strigose (vs. villous on both surfaces), and strigose veins (vs. villous).

Anemia $\times$ elephantensis N.T.L. Pena \& Schwartsb., nothosp. nov. Type: Brazil. Espírito Santo, Nova Venécia: Área de Preservação Ambiental Pedra do Elefante, $18^{\circ} 77^{\prime} 75^{\prime \prime S}, 40^{\circ} 43^{\prime} 35^{\prime \prime} \mathrm{W}, 300 \mathrm{~m}$, 20-IX-2015, N.T.L. Pena, P.B. Schwartsburd, C.V. Miranda \& J. Luber 649 (holotype: VIES).

Figures 3e-h

Plants terrestrial. Rhizomes decumbent, with brownish hairs. Fronds erect, $25-30 \mathrm{~cm}$ long; stipes $13-18 \mathrm{~cm} \times 0.5-1 \mathrm{~mm}$, brownish to greenish, adaxially sulcate, not winged, glabrescent to sparsely villous; fertile pinnae erects, $6-12 \mathrm{~cm}$ long, trespassing the sterile laminae portion. Laminae 1-pinnate to 2-pinnate, ovate to lanceate, with 9-11 pairs of pinnae, apex pinnatifid to subconform, $8 \times 5 \mathrm{~cm}$; rachises glabrescent to villous; basal pinnae entire, deeply dissected basioscopically forming one basal lobe, to 1-pinnate, ovate to lanceate, margins entire, apex rounded, $2-4 \times 0.5-1 \mathrm{~cm}$; costae abaxially sparsely villous, adaxially sparsely strigose; veins free, anastomosing toward pinna margins, strigose on both surfaces. Spores few but well-developed.

Distribution: known only from the type collection.

Etymology: the epithet refers to the place of occurrence where it was collected: Pedra do Elefante (=Elephant Stone). 


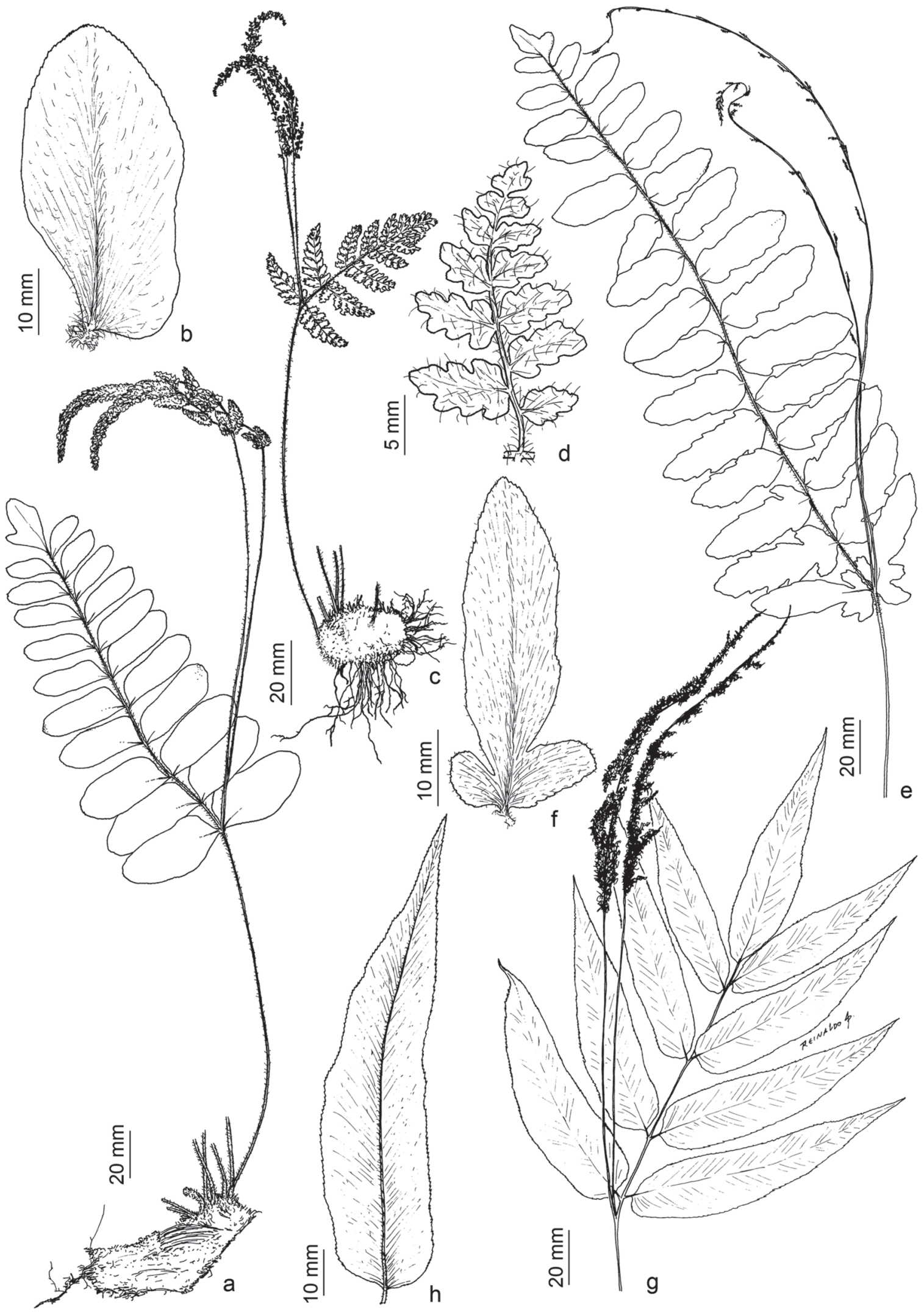

Figure. 2. a, b. Anemia collina (Pena 495). a. Habit. b. Basal pinna. c, d. A. ferruginea var. ferruginea (Pena 258). c. Habit. d. Basal pinna. e, f. A. organensis (Pena 369). e. Frond. f. Basal pinna. g, h. A. phyllitidis (Pena 500). g. Frond. h. Basal pinna. 
Comments: This putative new hybrid bears several shared morphological features between Anemia hirsuta and A. luetzelburgii (table 1).

According to Mickel (1982, 2016), hybridization involving Anemia hirsuta (tetraploid; $\mathrm{n}=76$ ) is common in the Neotropics. This author cites that various genera (such as Asplenium, Cystopteris and Doryopteris) present neopolyploidism, but Anemia has a higher level of ploidy, varying from $2 \mathrm{n}$ to $14 \mathrm{n}$. Those genetic features turn it into a favored group for hybridization. Anemia hirsuta, in particular, has reported events of hybridization involving another 12 species (Mickel \& Smith 2004, Mickel 2016, Rabelo $\&$ Schwartsburd 2016). The latter was not previously accounted in hybridization events.

Brade (1947) described a new hybrid for Espírito Santo state (Anemia xespiritosantensis Brade) and he believed that the probable parents were A. collina and $A$. tenella (Cav.) Sw. However, the images of " $A$. tenella" provided by Brade (1947) are, in our point of view, better identified as $A$. luetzelburgii. Thus, $A$. $\times$ espiritosantensis is very probably resulted from a cross of $A$. collina and $A$. luetzelburgii.

Anemia $\times$ elephantensis differs from $A$. $\times$ espiritosantensis by fronds ca. $25-30 \mathrm{~cm}$ long (vs. ca. $45 \mathrm{~cm}$ long), veins proximally free, but anastomosing toward pinna margins (vs. all veins free), and welldeveloped spores (vs. aborted spores or in uncommon formats).

Anemia ferruginea Humb. \& Bonpl. ex Kunth var. ferruginea, Nov. Gen. Sp. [H.B.K.] 1: 32. 1815.

Figures $2 \mathrm{c}, \mathrm{d}$

Plants rupestrial. Rhizomes short-creeping, with reddish hairs. Fronds erect, 19-40 cm long; stipes $10-26 \mathrm{~cm} \times 0.7-2 \mathrm{~mm}$, brownish, adaxially sulcate, not winged, densely villous; fertile pinnae erect, $7-11 \mathrm{~cm}$ long, not trespassing the sterile laminae portion. Laminae 2-pinnate-pinnatifid, lanceate or lanceolate, 8-15 pairs of pinnae, apex pinnatifid to subconform, $5-13 \times 2-5 \mathrm{~cm}$; raques densely villous; basal pinnae 1-pinnate-pinnatifid, ovate to lanceate, margins entire or crenate, apex acute to rounded, $1.5-3 \times 0.5-1 \mathrm{~cm}$; costae villous on both surfaces; veins free, abaxially sparselly villous, adaxially villous; sessile glands present. Spores well-developed.

Distribution: Colombia, Venezuela, Guyana, Ecuador, Peru, Brazil and Bolivia. In Brazil is reported to north (Amazonas, Roraima), northeast (Bahia, Maranhão), middle-western (Goiás, Mato Grosso), southeast
(Minas Gerais, São Paulo) and south (Paraná) regions (Mickel 2016).

Specimens examined: afloramento rochoso da Dona Ecília, 1877'44"S, 4046'38"W, 448 m, 05-V-2015, N.T.L. Pena et al. 258 (VIC; VIES).

Additional specimens examined: Brazil; Minas Gerais; Parque Estadual de Serra do Brigadeiro; Serra das três Cabeças, Totem Deitado, 2068'81"S, 42 ${ }^{\circ} 45^{\prime} 47^{\prime \prime} \mathrm{W}, 16-\mathrm{VI}-2003$, G.E. Valente \& R.M.S.A. Meira 1432 (VIC).

Comments: Anemia ferruginea is commonly identified as two varieties: A. ferruginea var. ferruginea and A. ferruginea var. ahenobarba (Christ) Mickel (not found in the study area). They can be distinguished by laminae more dissected with acute or acuminate apex in A. ferruginea var. ahenobarba (e.g. Mickel 1962, Casarino 2009). Among the analyzed species, A. ferruginea var. ferruginea can be recognized by laminae 2-pinnate-pinnatifid and apex pinnatifid to subconform.

Anemia hirsuta (L.) Sw., Syn. Fil. (Swartz) 156. $1806 \equiv$ Osmunda hirsuta L., Sp.P1. 2: 1064. 1753. Figures 3a,b

Plants terrestrial or rupestrial. Rhizomes decumbent, orange hairs. Fronds erect, $23-47 \mathrm{~cm}$ long; stipes $13-23 \mathrm{~cm} \times 0.6-1 \mathrm{~mm}$, brownish, adaxially sulcate, not winged, glabrescent to villous; fertile pinnae erect, $10-21 \mathrm{~cm}$ long, always trespassing the sterile laminae portion. Laminae 1-pinnate-pinnatifid, ovate to lanceolate, 8-11 pairs of pinnae, apex pinnatifid to subconform, 6-11 $\times 2-4 \mathrm{~cm}$; rachises glabrescent to sparselly villous; basal pinnae entire, irregularly dissected, with 1-3 pairs of lobes, ovate to oblong, margins entire to crenate, apex rounded, $1-2 \times 0.5-1 \mathrm{~cm}$; costae glabrescent to sparsely villous on both surfaces; veins free, glabrescent to villous on both surfaces. Spores well-developed.

Distribution: Mexico, Guatemala, Honduras, HI Salvador, Nicaragua, Costa Rica, Panama, Greater Antilles, Trinidad \& Tobago, Colombia, Venezuela, Ecuador, Peru, Brazil and Bolivia. In Brazil is reported to northeast (Bahia, Piauí), middle-western (Goiás, Maranhão, Mato Grosso) and southeast (Minas Gerais, Rio de Janeiro, São Paulo) regions (Mickel 2016).

Specimens examined: Estrada para carro em direção a Pedra do Elefante, $18^{\circ} 77^{\prime} 75^{\prime \prime} \mathrm{S}, 40^{\circ} 43^{\prime} 35^{\prime \prime} \mathrm{W}, 327 \mathrm{~m}$, 20-IX-2015, N.T.L. Pena et al. 650 (VIES); Serra de Baixo, 18 46'37"S, 40²6'38"W, 313 m, 18-I-2008, 
P.H. Labiak et al. 4677 (MBML; CEPEC; UPC); Fazenda Santa Rita, $18^{\circ} 46^{\prime} 38^{\prime \prime} \mathrm{S}, 40^{\circ} 27^{\prime} 40^{\prime \prime} \mathrm{W}, 300 \mathrm{~m}$, 13-I-2009, P.H. Labiak et al. 5075 (MBML; CEPEC; UPCB).

Additional specimens examined: Brasil; Minas Gerais; Viçosa; Campus da UFV, pedreira da caprinocultura, $20^{\circ} 76^{\prime} 43^{\prime \prime S}, 42^{\circ} 86^{\prime} 42^{\prime \prime} \mathrm{W}, 12-X I I-1996$, G.E. Valente et al. 241 (VIC); idem, G.E. Valente et al. 242 (VIC).

Comments: Anemia hirsuta presents 1-pinnatepinnatifid laminae, which are ovate to lanceate, and free veins. The basal pinnae are pinnatifid, ovate to oblong, with entire to crenate margins (see also the comments of $A$. ×elephantensis).

Anemia luetzelburgii Rosenst. Repert. Spec. Nov. Regni Veg. 20: 94. 1924.

Figures $3 \mathrm{c}, \mathrm{d}$

Plants terrestrial or rupestrial. Rhizomes erect to decumbent, with brownish to orange hairs. Fronds erects, 26-40 cm long; stipes $15-30 \mathrm{~cm} \times 0.7-1.5 \mathrm{~mm}$, brownish to greenish, adaxially sulcate, not winged, sparsely villous to glabrescent; fertile pinnae erect, $10-20 \mathrm{~cm}$ long, trespassing or not the sterile laminae portion. Laminae 1-pinnate to 2-pinnate, deltade, 10-14 pairs of pinnae, apex pinnatifid to subconform, $11-17 \times 11-17 \mathrm{~cm}$; rachises glabrescent to villous; basal pinnae 1-pinnate, lanceate, margins entire to crenate, apex acute, 3-9 $\times 1-3 \mathrm{~cm}$; costae sparsely strigose on both surfaces; veins free, anastomosing toward pinna margins; strigose on both surfaces. Spores well-developed.

Distribuition: endemic to Brazil, reported to northeast (Bahia) and southeast (Espírito Santo, Minas Gerais, Rio de Janeiro) regions (Mickel 2016).

Specimens examined: estrada para carro em direção à Pedra do Elefante, $18^{\circ} 77^{\prime} 75^{\prime \prime} \mathrm{S}, 40^{\circ} 43^{\prime} 35^{\prime \prime} \mathrm{W}, 330 \mathrm{~m}$, 18-IX-2015, N.T.L. Pena et al. 439 (VIC; VIES); Trilha Pedra do Elefante, $18^{\circ} 76^{\prime} 38^{\prime \prime} \mathrm{S}, 40^{\circ} 44^{\prime} 85^{\prime \prime}, 308 \mathrm{~m}$, 19-IX-2015, N.T.L. Pena et al. 469 (VIC; VIES); Cachoeira da $\mathrm{D}^{\mathrm{a}}$ Ecília, $18^{\circ} 46^{\prime} 45^{\prime \prime} \mathrm{S}, 40^{\circ} 25^{\prime} 57^{\prime \prime} \mathrm{W}$, 138 m, 20-IX-2015, N.T.L. Pena et al. 501 (VIC; VIES); idem, N.T.L. Pena et al. 505 (VIC; VIES); Afloramento Rochoso Pedra do Elefante, 18 $46^{\prime} 30^{\prime \prime} \mathrm{S}$, 40²7'42”W, 604 m, 7-III-2016, N.T.L. Pena et al. 599 (VIC; VIES); Serra de baixo, afloramento rochoso na base da Preda do elefante, $18^{\circ} 45^{\prime} 00^{\prime \prime} \mathrm{S}, 40^{\circ} 27^{\prime} 00^{\prime \prime} \mathrm{W}$, 291 m, 9-V-2008, C.N. Fraga et al. 2073 (RB - Photo!)

Additional specimens examined: Brazil; Espirito Santo, Cariacica, Reserva Biológica duas Bocas, 

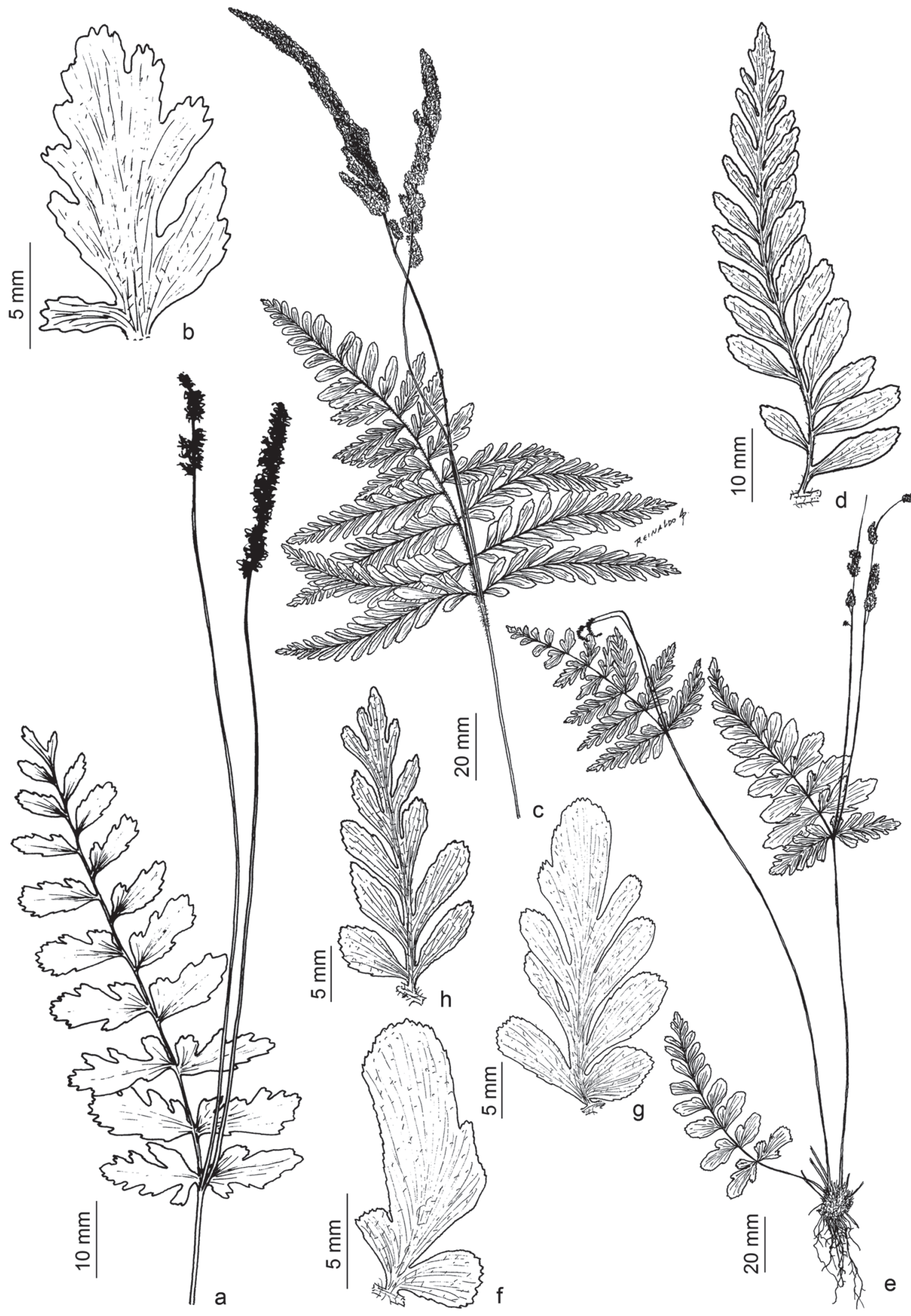

Figure. 3. a, b. Anemia hirsuta (Valente 241). a. Frond. b. Basal pinna. c, d. A. luetzelburgii (Pena 439). c. Frond. d. Basal pinna. e-h. A. ×elephantensis (Pena 649 - holotype). e. Habit. f-h. Basal pinnae. 
$20^{\circ} 16^{\prime} 22^{\prime}$ S, 40²8'36”W, 200m, 16-I-2009, P.H. Labiak et al. 5144 (RB - photo!).

Comments: Anemia luetzelburgii was commonly found in Pedra do Elefante and it can be terrestrial or rupestrial. This species has free veins in the proximal portions, but anastomosing toward pinna margins. This latter feature is shared with A. xelephantensis.

Anemia organensis Rosenst., Repert. Spec. Nov. Regni Veg. 20: 95. 1924.

Figures 2e, f

Plants terrestrial or rupestrial. Rhizomes shortcreeping, with brownish hairs. Fronds erects, $30-60 \mathrm{~cm}$ long; stipes $12-24 \mathrm{~cm} \times 1.3-2.5 \mathrm{~mm}$, brownish to greenish, adaxially sulcate, not winged, glabrescent to villous; fertile pinnae erect, $18-32 \mathrm{~cm}$ long, not trespassing the sterile laminae portion. Laminae 1-pinnate to 1-pinnate-pinnatifid, lanceate, 10-18 pairs of pinnae, apex subconform, 19-27 × 6-10 cm; rachises densely villous; basal pinnae entire, dissected basioscopically forming two basal lobes, oblong, margins entire, apex round, $4-5 \times 1-1.5 \mathrm{~cm}$; costae with both surfaces densely villous; veins free, villous on both surfaces. Spores well-developed.

Distribution: endemic to Brazil, reported to northeast (Bahia) and southeast (Espírito Santo, Rio de Janeiro) regions (Mickel 2016).

Specimens examined: afloramento Rochoso Pedra do Elefante, $18^{\circ} 46^{\prime} 30^{\prime \prime} \mathrm{S}, 40^{\circ} 27^{\prime} 42^{\prime \prime} \mathrm{W}, 568 \mathrm{~m}$, 25-III-2015, N.T.L. Pena et al. 155 (VIC; VIES); idem, $18^{\circ} 46^{\prime} 27^{\prime \prime S}, 40^{\circ} 27^{\prime} 42^{\prime \prime}, 540 \mathrm{~m}, 27-\mathrm{VII}-2015$, N.T.L. Pena et al. 369 (VIES); idem, 18 46'24"S, 40²7'42", 600 m, 7-III-2016, N.T.L. Pena \& A. Alves-Araújo 600 (VIES); idem, 18 46' 12"S, 40²7'27"W, 26-IV-2010, A.M. Assis et al. 2491 (MBML); Afloramento rochoso à direita na subida para a Pedra do Elefante, $18^{\circ} 46^{\prime} 13^{\prime \prime S}, 40^{\circ} 26^{\prime} 51^{\prime \prime} \mathrm{W}, 300-600$ m, 14-I-2009, P.H. Labiak et al. 5110 (MBML; CEPEC; RB; UPCB); Serra de Baixo, $18^{\circ} 46^{\prime} 10^{\prime \prime} \mathrm{S}, 40^{\circ} 27^{\prime} 45^{\prime \prime} \mathrm{W}, 420-653 \mathrm{~m}$, 19-II-2008, A.P. Fontana et al. 4872 (MBML; UPCB; $\mathrm{RB}$ ); idem, $18^{\circ} 46^{\prime} 10^{\prime \prime} \mathrm{S}, 40^{\circ} 27^{\prime} 28^{\prime \prime} \mathrm{W}, 630 \mathrm{~m}, R$. Goldenberg et al. 1148 (MBML; RB).

Comments: specimens of Anemia organensis can be found as terrestrial or rupestrial. This taxon is easily recognized by the presence of short-creeping rhizomes, 1-pinnate laminae, and pinnae with entire margins and rounded apex. See morphological comparisons with $A$. collina in its comments.
Anemia patens Mickel \& Labiak in Mickel, Fl. Neotrop. Monogr. 118: 68, figs. 62A-C. 2016. Figures $4 \mathrm{e}, \mathrm{f}$

Plants rupestrial. Rhizomes short-creeping, with brownish hairs. Fronds erect, 35-40 cm long; stipes $15-20 \mathrm{~cm} \times 2-3 \mathrm{~mm}$, stramineous, adaxially sulcate, not winged, glabrescent to villous; fertile pinnae erect, $20-22 \mathrm{~cm}$ long, generally trespassing the sterile laminae portion. Laminae 2-pinnate-pinnatifid to 3-pinnate, ovate to lanceolate, 12-15 pairs pinnae, apex subconform, 15-20 × 15-18 cm; rachises densely vilous; basal pinnae 1-pinnate-pinatifid, ovate lanceolate, margins entire, apex rounded, $8 \times 4 \mathrm{~cm}$; costae villous on both surfaces; veins free, abaxially villous, adaxially sparselly villous; sessile glands present. Spores well-developed.

Distribuition: endemic to Brazil, reported to southeast (Espírito Santo) region (Mickel 2016).

Specimes examined: Serra de Baixo, 18 46'58"S, 40²6'47"W, 420m, 18-II-2008, P.H. Labiak et al. 4689 (MBML; CEPEC; RB; UPCB).

Comments: this taxon is morphologically similar to Anemia tomentosa var. tomentosa and A. tomentosa var. anthriscifolia, however Anemia patens presents laminae (2-pinnate-pinnatifid to 3-pinnate), and basal pinnae with rounded apices.

Anemia phyllitidis (L.) Sw., Syn. Fil. (Swartz) 155. $1806 \equiv$ Osmunda phyllitidis L., Sp.P1.2: 1064. 1753. Figures $2 \mathrm{~g}, \mathrm{~h}$

Plants terrestrial. Rhizomes erect to decumbent, with dark brown to orange hairs. Fronds erect, 40-48 cm long; stipes $20-32 \mathrm{~cm} \times 1.2-3.2 \mathrm{~mm}$, brownish to greenish, adaxially sulcate, not winged, glabrescent to villous; fertile pinnae erect, $20-25 \mathrm{~cm}$ long, generally trespassing the sterile laminae portion. Laminae 1-pinnate, ovate to lanceolate, 6-7 pairs of pinnae, apex subconform, 15-23 × 13-17 cm; rachises glabrescent to sparselly villous; basal pinnae entire, ovate to lanceate, margins crenate to dentate, apex acute, 6-9 $\times 2-3 \mathrm{~cm}$; costae glabrescent to sparselly villous on both surfaces; veins anastomosed, glabrescent to sparselly strigose on both surfaces. Spores well-developed.

Distribution: Mexico, Guatemala, Honduras, El Salvador, Nicaragua, Costa Rica, Panama, Greater Antilles, Colombia, Venezuela, Trinidad, Guyana, Suriname, Ecuador, Peru, Brazil, Bolivia, Paraguay, Argentina, Uruguay (Mickel 2016). 

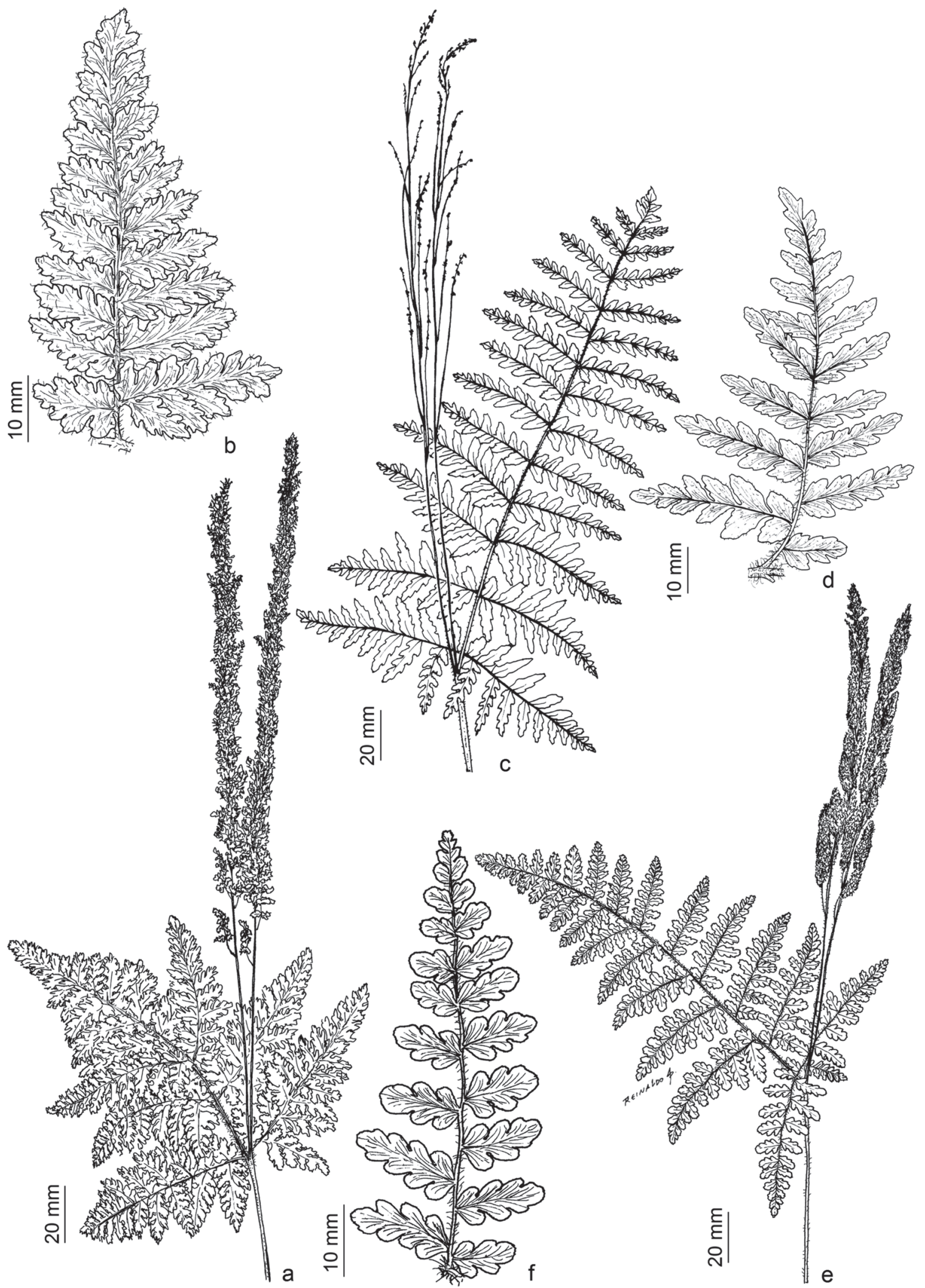

Figure. 4. a, b. Anemia tomentosa var. anthriscifolia (Pena 507). a. Frond. b. Basal pinna. c, d. A. tomentosa var. tomentosa (Pena 589). c. Frond. d. Basal pinna. e, f. A. patens (Labiak 4689). e. Frond. f. Basal pinna. 
Specimens examined: Trilha da $\mathrm{D}^{\mathrm{a}}$ Ecília, $18^{\circ} 78^{\prime} 25^{\prime \prime S}$, 4043'61"W, 195 m, 16-III-2015, N.T.L. Pena et al. 107 (VIES); idem, N.T.L. Pena et al. 108 (VIC; VIES); Trilha da cachoeira $\mathrm{D}^{\mathrm{a}}$ Ecília, $18^{\circ} 46^{\prime} 45^{\prime \prime} \mathrm{S}$, $40^{\circ} 25^{\prime} 57^{\prime \prime} \mathrm{W}, 142 \mathrm{~m}, 19-\mathrm{XI}-2015$, N.T.L. Pena et al. 500 (VIC; VIES).

Additional specimens examined: Brasil, Minas Gerais, Parque Estadual do Rio Doce, trilha da capolina, 2-V-1996, D. Graçano 149 (VIC); Rio Doce, trilha do Porto Capim, 2-V-1996, D. Graçano 161 (VIC); Viçosa, UFV, 2046'18"S, 4251'06"W, $780 \mathrm{~m}$, 29-I-2015, Schwartsburd et al. 3477 (VIC).

Comments: Anemia phyllitidis is easily identified by presenting erect to decumbent rhizomes, laminae 1-pinnate, pinnae with crenate to dentate margins and acute apex. Anemia phyllitidis is the only taxon with fully anastomosing veins, from Pedra do Elefante. Rabelo \& Schwartsburd (2016) found a hybrid between A. phylitidis and A. hirsuta: A. × semihirsuta Mickel. This hybridization event is common and was also reported for Mexico (Mickel \& Smith 2004). We did not find it in Pedra do Elefante, even though both parents are present there.

Anemia tomentosa (Sav.) Sw. var. tomentosa, Syn. Fil. (Swartz) 157. 1806 $\equiv$ Osmunda tomentosa Sav. in Lam., Encycl. 4: 652. 1797.

Figures 4c,d

Plants terrestrial or rupestrial. Rhizomes shortcreeping, with brownish to orange hairs. Fronds erect, 40-70 cm long; stipes $27-35 \mathrm{~cm} \times 1.2-3.5 \mathrm{~mm}$, dark brown, adaxially sulcate, not winged, glabrescent to villous; fertile pinnae erect, $12-30 \mathrm{~cm}$ long, generally trespassing the sterile laminae portion. Laminae 2-pinnate to 2-pinnate-pinnatifid, ovate to lanceolate, 9-13 pairs of pinnae, apex pinnatifid, $10-17 \times 10-15 \mathrm{~cm}$; rachises densely villous; basal pinnae 1-pinnate-pinnatifid, ovate to lanceolate, margins entire, apex acute, $8-11 \times 4-6 \mathrm{~cm}$; costae villous on both surfaces; veins free, villous on both surfaces; sessile glands present. Spores aborted.

Distribution: Brazil, Paraguay, Northeast of Argentina and Uruguay. In Brazil is reported to southeast (Espírito Santo, Rio de Janeiro) and south (Paraná, Rio Grande do Sul, Santa Catarina) regions (Mickel 2016).

Specimens examined: Trilha da $\mathrm{D}^{\mathrm{a}}$ Ecília, $18^{\circ} 76^{\prime} 54^{\prime \prime} \mathrm{S}$, 4044'16"S, 284 m, 9-IV-2015, N.T.L. Pena \& J. Luber 185 (VIES); Estrada para Pedra do Elefante, $18^{\circ} 46^{\prime} 16^{\prime \prime} \mathrm{S}, 40^{\circ} 27^{\prime} 55^{\prime \prime} \mathrm{W}, 300 \mathrm{~m}, 21-\mathrm{XII}-2015$,
N.T.L. Pena et al. 589 (VIES); Afloramento rochoso a direita da Pedra do Elefante subindo, 18 ${ }^{\circ} 46^{\prime} 32^{\prime \prime} \mathrm{S}$, 40²7'02"W, $450 \mathrm{~m}, 8$-III-2016, N.T.L. Pena \& A. Alves-Araújo 635 (VIC; VIES); Serra de Baixo $18^{\circ} 46^{\prime} 58^{\prime \prime} \mathrm{S}, 40^{\circ} 26^{\prime} 47^{\prime \prime} \mathrm{W}, 420 \mathrm{~m}, 18-\mathrm{II}-2008$, P.H. Labiak et al. 4687 (MBML; CEPEC; RB; UPCB).

Additional specimens examined: Brasil; Minas Gerais; Viçosa; Campus UFV. Floresta estacional Semi-Decidual. P.H. Rolfs, próximo a restaurante Universitário, 20-I-2014, P.B. Schwartsburd et al. 2926 (VIC).

Comments: Anemia tomentosa var tomentosa differs from $A$. tomentosa var anthriscifolia by having fronds with ca. 40-70 cm long (vs. ca. 25-40 cm long), petioles up to ca. $3.5 \mathrm{~mm}$ in diam. (vs. ca. $1-2 \mathrm{~mm}$ ) and aborted spores (vs. well-developed). This is the first record of this variety for Espírito Santo State, which was previously known only to Minas Gerais, Rio de Janeiro, São Paulo, Paraná, Santa Catarina, Rio Grande do Sul, Paraguay, northeast of Argentina, and Uruguay (Mickel 2016, Rabelo \& Schwartsburd 2016).

Anemia tomentosa var. anthriscifolia (Schrad.) Mickel, Iowa State J. Sci. 36: 424. $1962 \equiv$ Anemia anthriscifolia Schrad. Gott. gel. Anz. 1824.

Figures $4 a, b$

Plants terrestrial or rupestrial. Rhizomes shortcreeping, with brownish to orange hairs. Fronds erect, 25-40 cm long; stipe (3-)10-24 cm $\times 1-2 \mathrm{~mm}$, dark brown, adaxially sulcate, not winged, glabrescent to densely villous; fertile pinnae erect, 17-26 cm compr., trespassing the sterile laminae portion. Laminae 2-pinnate-pinnatifid, ovate to lanceolate, 12-14 pairs of pinnae, apex pinnatifid, $8-10 \times 5-8 \mathrm{~cm}$; rachises densely villous; basal pinnae 1-pinnate-pinnatifid, ovate to lanceolate, margins entire, apex acute, $3-4 \times 1-2 \mathrm{~cm}$; costae villous on both surfaces; veins free, villous on both surfaces, sessile glands present. Spores well-developed.

Distribution: Brazil, Bolivia, Paraguay and North of Argentina. In Brazil is reported to northeast (Bahia, Paraíba, Pernambuco), middle-western (Mato Grosso), southeast (Espírito Santo, Minas Gerais, Rio de Janeiro, Sao Paulo) and south (Rio Grande do Sul) regions (Mickel 2016).

Specimens examined: afloramento rochoso a esquerda de Pedra do Elefante na subida, $18^{\circ} 77^{\prime} 45^{\prime \prime} \mathrm{S}$, 4046'06"W, 18-VI-2015, N.T.L. Pena \& W.O. Souza 
284 (VIES); idem, N.T.L. Pena \& W.O. Souza 285 (VIES); idem, 1877'45"S, 4046'06"W, 495 m, 18IX-2015, N.T.L. Pena et al. 507 (VIC; VIES); Estrada para carro em direção a Pedra do Elefante, $18^{\circ} 77^{\prime} 75^{\prime \prime} \mathrm{S}$, 4043'35"W, 213 m, 20-IX-2015, N.T.L. Pena et al. 488 (VIC; VIES); Afloramento rochoso $\mathrm{D}^{\mathrm{a}}$ Ecília, $18^{\circ} 77^{\prime} 44^{\prime \prime S}, 40^{\circ} 46^{\prime} 38^{\prime \prime W}, 400$ m, 7-III-2016, N.T.L. Pena \& A. Alves-Araújo 592 (VIC; VIES); Serra de Baixo, $18^{\circ} 48^{\prime} 00^{\prime \prime S}, 40^{\circ} 28^{\prime} 00^{\prime \prime} \mathrm{W}, 9-\mathrm{V}-2008$, C.N. Fraga et al. 2080 (MBML; CEPEC; RB; UPCB); Fazenda Santa Rita, $18^{\circ} 46^{\prime} 56^{\prime \prime} \mathrm{S}, 40^{\circ} 26^{\prime} 90^{\prime \prime} \mathrm{W}$, 26-III-2009, A.M. Assis et al. 2362 (MBML); Trilha principal da mata da fazenda Santa Rita, $18^{\circ} 46^{\prime} 67^{\prime \prime} \mathrm{S}$ 4025'58" W, 154 m, 16-VIII-2008, A.M.A Amorim et al. 7502 (RB - Photo!).

Comments: see comments of $A$. tomentosa var. tomentosa.

\section{Acknowledgments}

The senior author thanks Fundação de Amparo à Pesquisa do Espírito Santo (FAPES) for the fellowship. All authors would like to thank IEMA for the license granted, Instituto Nacional da Mata Atlântica for permission of using data and equipments, MBML and VIC herbaria staff. We also thank Paulo H. Labiak for providing information about Anemia patens, and everybody from the Laboratory of Sistemática e Genética Vegetal (SGV)/CEUNES/UFES.

\section{Literature cited}

Brade, A.C. 1974. Contribuição para o conhecimento da flora do estado do Espírito Santo (I. Pteridophyta). Rodriguésia 21: 25-56.

Casarino, J.E, Mynssen, C.M. \& Messias, M.C.T.B. 2009. Schizaeales no Parque Estadual do Itacolomi, Minas Gerais, Brasil. Revista Brasileira de Botânica 32: 737-740.

IBGE (Instituto Brasileiro de Geografia e Estatística). 2012. Instituto Brasileiro de Geografia e Estatística. Manual Técnico da Vegetação Brasileira. Série Manuais Técnicos em Geociências, 2 ed. IBGE, Rio de Janeiro.

INCAPER (Instituto Capixaba de Pesquisa, Assistência Técnica e Extensão Rural). 2017. Hidrometeriologia. Avaiable in http://hidrometeorologia.incaper.es.gov.br (access in 15-VI- 2017).

Labiak, P.H., Mickel, J.T. \& Hanks, J.G. 2015. Molecular phylogeny and character evolution of Anemiaceae (Schizaeales). Taxon 64: 1141-1158.
Lellinger, D.B. 2002. A Modern Multilingual Glossary for Taxonomic Pteridology. American Fern Society, Inc.

Mickel, J.T. 1962. A monographic study of fern genus Anemia, suggenus Coptophyllum. Iowa State College Journal of Science 36: 349-492.

Mickel, J.T. 1982. The genus Anemia (Schizaeaceae) in Mexico. Brittonia 34: 388-413.

Mickel, J.T. 2016. Anemia (Anemiaceae). Flora Neotropica Monograph 118: 1-181.

Mickel, J.T. \& Smith, A.R. 2004. The pteridophytes of Mexico. Memoirs of the New York Botanical Garden 88: 1-1055.

Moran, R.C \& Mickel, J.T. 1995. Anemia Sw. In: R.C. Moran, R. Riba (eds. for pteridophytes), Psilotaceae a Salviniaceae. In: G. Davidse, M. Sousa, S. Knapp (general eds.). Flora Mesoamericana,v. 1. Universidad Nacional Autónoma de México, Mexico City, pp. 53-56.

Myers, N., Mittermeier, R.A., Mittermeier, C.G., Fonseca, G.A.B. \& Kent, J. 2000. Biodiversity hotspots for conservationpriorities. Nature 403: 853-858.

PPG I (Pteridophyte Phylogeny Group). 2016. A community-derived classification for extant lycophytes and ferns. Journal of Systematics and Evolution 54: 563-603.

Porembski, S. 2000. The invasibility of tropical granite outcrops ('inselbergs') by exotic weeds. Journal of the Royal Society of Western Australia 83:131-137.

Prado, J., Sylvestre, L.S., Labiak, P.H., Windisch, P.G., Salino, A., Barros, I.C.L., Hirai, R.Y., Almeida, T.E., Santiago, A.C.P., Kieling-Rubio, M.A., Pereira, A.F.N., Øllgaard, B., Ramos, C.G.V., Mickel, J.T., Dittrich, V.A.O., Mynssen, C.M., Schwartsburd, P.B., Condack, J.P.S., Pereira, J.B.S. \& Matos, F.B. 2015. Diversity of ferns and lycophytes in Brazil. Rodriguésia 66: 1-11.

Rabelo, L.S. \& Schwartsburd, P.B. 2016. Schizaeales (Filicopsida, Tracheophyta) of Viçosa, Minas Gerais, Brazil, with special reference to hybrids. Brittonia 68: 379-396.

Thiers, B. 2017. [continuously updated]. Index Herbariorum: A global directory of public herbaria and associated staff. New York Botanical Garden's Virtual Herbarium. Available in http://sweetgum.nybg. org/science/ih/ (acess in 15-VI-2017).

Tryon, R.M. 1972. Endemic areas and speciation in tropical American ferns. Biotropica 4: 76-84.

Windisch, P.G. 1992. Pteridófitas da região norte-ocidental do Estado de São Paulo: guia para estudo e excursões. 2 ed. Universidade Estadual Paulista, São José do Rio Preto. 